\title{
Sequential and global optimization in powder injection molding processing
}

\author{
Ghassane Ayad* — Thierry Barriere* — Jean-Claude Gelin* \\ Jiupeng Song*,** — Baosheng Liu** \\ * Femto-ST Institute, Department of Applied Mechanics, ENSMM Besançon \\ 24 Rue de l'Epitaphe, 25000, Besançon, France \\ \{jean-claude.gelin, jiupeng.song, thierry.barriere\}@ens2m.fr \\ ** Department of Applied Mechanics, Southwest Jiaotong University \\ 610031, Chengdu, Sichuan, P. R. China
}

\begin{abstract}
The work is focused on the overall optimization associated to powder injection molding (PIM). The PIM process includes four main stages, from the mixture of the powders and binders to the final sintering stage. Injection and sintering stages are considered to be the most important for optimization, as they mostly affect the final quality of the produced components. The injection stage shapes the green parts but initiates powders segregation that will be inherited and amplified by the sintering stage to finally appear in the resulting products. One first introduces an optimization loop based on the surfaces response method to minimize the powder segregation. Then the results are transferred to a sintering optimization loop applied through an experimentally calibrated thermo-mechanical creep model to predict the shrinkage and density contours on the final parts. The overall optimization combines both optimizers based on the developed simulation tools to provide a realistic way to improve the PIM process design accounting the different processing stages.

RÉSUMÉ. Un modèle continu basé sur l'évolution d'une loi de comportement visco-plastique est utilisé pour décrire le procédé de densification par diffusion en phase solide pour des composants fabriqués par le procédé de moulage par injection de poudres céramiques CIM. Une méthode d'optimisation est proposée afin de minimiser la ségrégation pour l'étape de moulage et d'identifier les paramètres du modèle de la loi de comportement pour l'étape de densification. La simulation de l'optimisation globale d'une prothèse de hanche est réalisée par ce procédé CIM et pris comme exemple du point de vue numérique et expérimental afin de minimiser la ségrégation, de prédire les retraits et les dimensions de la cavité de l'empreinte du moule. Les résultats numériques sont validés par des essais expérimentaux. KEYWORDS: powder injection molding, processing stages, sintering, overall optimization. MOTS-CLÉS: moulage par injection de poudres, densification, optimisation séquentielle et globale.
\end{abstract}

DOI:10.3166/REMN.17.843-855 @ 2008 Lavoisier, Paris 


\section{Introduction}

The material processing by powder injection molding (PIM) exhibits important advantages for the manufacturing of small components in 3D complicated shapes (German, 1997). The overall PIM process includes four main stages. Firstly the feedstock composed of a mixture of powders and binders should be prepared. Then the original shape of the component is obtained by powder injection molding. The third step consists to remove the binder by thermal or catalytic debinding processes. The final product results from the last sintering stage, in which the porous material is transformed to a full dense one by the solid state sintering process. The quality of the final parts results from their shape and size accuracy, as well as the respect of the required mechanical properties. The eventual defects that start to occur in the injection stage are accentuated in the next debinding and sintering stages. These defects may be effectively amplified by the sintering process resulting in shape distortions and improper mechanical properties in the final products (Barriere, 2005). The complete control of final quality requires multistage optimization for both the individual stages and their sequential effects in the whole PIM process. The original feedstock injected into the mould is considered as homogeneous resulting from sufficient mixing of the powder and binder components. The processing defects begin to appear during the injection stage, associated to the filling flow in sprues, runners and inlets associated to mould cavities, as both the geometrical aspects and the behavior of mixture flows. Besides other defects that can be observed or measured, the powder segregation is difficult to monitor after the injection. If the segregation is important, it will result in significant distortions in the remaining debinding and sintering stages. However, even if the green parts after injection seem perfect, they may include important segregation. One may evaluate segregation effects induced in the early injection stage only after the sintering stage. So the optimization of PIM process is then an indirect one, which requires the optimization of the subsequent stages with the transport of important data and finally overall optimization of the whole process.

The segregation in the injection stage is evaluated through the numerical simulation based on a biphasic model (Gelin, 2004). The powder and binder volume fraction phases are expressed by two field variables, which stand for the quantity of powder segregation during and after the injection process (Barriere, 2001). It is generally supposed that distribution of the powder density is not effectively changed in the next debinding stage. The role of debinding is mainly to remove the binder components resulting in change in shape of the green parts. At the microscale, powder particles remain in similar state, except that the binder between them is almost removed. The effect of inhomogeneous powders distribution in sintering is evident. It is in this stage that the early induced powder segregation exhibits its important effects on quality of the resulting products.

The sintering process is evaluated through simulation based on a viscoplastic creep model. This model is based on phenomenological principles and identified 
through specially designed experiments. The sintering process inherits the density distribution that results from injection molding simulation, with the subtraction of binder phase due to debinding process. Such a simulation based on the calibrated model provides the determination of shrinkage ratios, shape distortions and final mechanical properties of the considered parts (Song, 2006).

The proposed optimization procedure includes the sequential optimization of the injection and sintering stages, with the binder removal from components between both stages. The overall optimization cycle for both processing stages is important too, allowing the optimization of overall parameters for the design of PIM processing. The validation of the optimization procedure is carried out considering that the PIM process is applied to a hip implant. This typical part is significant in terms of thickness and complicated in 3D shape. The parts have been manufactured in our laboratory through ceramic injection molding.

\section{Optimization of the injection stage}

\subsection{Powder segregation evaluation}

Besides other visible defects, such as jetting, front welding and incomplete filling, segregation induced by injection molding represents the most important defects that affect final quality of the products (German, 1997). In order to evaluate the segregation effects, a biphasic flow model has been developed to describe the mixture flow and to properly account powder segregation (Gelin, 2004). The flows of the powder and binder are respectively described by two distinct phases. The interaction between the flows of both phases is accounted through a term that represents their momentum exchange. The volume fraction field for the powder phase describes the segregation effects. The density distribution of the powder phase, as a function of the powder volume fraction, represents the result of segregation. For performing efficient simulation based on the proposed model, the Feapim ${ }^{\odot}$ FEM software has been developed by the authors based on the algorithms proposed in (Barriere, 2001, 2005).

\subsection{Optimization of segregation resulting from powder injection moulding}

Based on the developed finite elements simulation tools, the optimization of the segregation effects in injection molding was developed (Ayad, 2006). The objective function chosen for the optimization consists to minimize the gradient of the powder volume fraction in green parts. This objective function is expressed as:

$$
\left\{\begin{array}{l}
\min _{x} F_{O B J}(x) \\
\left(x_{i \min } \leq x_{i} \leq x_{i \max }\right)_{i=1, \ldots, 4}
\end{array} \quad \text { with } F_{O B J}(x)=\left(\frac{1}{N} \sum_{i=1}^{N} \frac{\left|\Phi_{S, i}(x)-\Phi_{S_{0}}\right|^{q}}{\Phi_{S_{0}}}\right)^{1 / q}\right.
$$


where $\Phi_{S_{0}}$ is the initial powder volume fraction, $N$ stands for total nodes number in the FEM mesh, and $\Phi_{S, i}$ stands the powder volume fraction at the $\mathrm{i}_{\mathrm{th}}$ node, $q$ is chosen equal to 1 . The optimization vector variable is $x=\left(x_{i}\right)_{i=1, \ldots, 4}$ Each component of the vector $x$ represents a parameter in the injection process, as indicated in Table 1. The optimization scheme is then formulated as the minimization of function [1] according to the optimization of 4 variables, under the constraints associated to the injection process, as related in Table 1.

Table 1. The optimization variables for the injection stage in PIM

\begin{tabular}{cll}
\hline Component & \multicolumn{1}{c}{ Parameters } & \multicolumn{1}{c}{ Optimized range } \\
\hline $\mathrm{x}_{1}$ & Powder volume fraction & {$[0.4-0.7]$} \\
\hline $\mathrm{x}_{2}$ & Interaction coefficient & {$\left[0.001-0.007 \mathrm{~Pa} \cdot \mathrm{s} \cdot \mathrm{m}^{-2}\right]$} \\
\hline $\mathrm{x}_{3}$ & Powder density & {$\left[1-8 \mathrm{~g} \cdot \mathrm{cm}^{-3}\right]$} \\
\hline $\mathrm{x}_{4}$ & Binder density & {$\left[0.5-2.5 \mathrm{~g} . \mathrm{cm}^{-3}\right]$} \\
\hline
\end{tabular}

The evaluation of the objective function with respect of the constraint functions leads to an expensive computational cost due the complexity of biphasic flows. Even if an efficient solver has been developed and used with success, it is still far away from the application of the FEM solver directly in the optimization loop. So, a response surface is built based on a limited number of the response function evaluations for the purpose to replace the FEM solver in optimization procedure. An adaptive refinement of the optimized space is associated to the application of the response surface. The optimization strategy is based on the use of a genetic algorithm that requires a large number of evaluations. So, in order to decrease the number of the cost function evaluations, an adaptative response surface is built. The nodes of the response surface function are assigned by the FEM solver. This approximate surface is stretched along dimensions of its variables. However an important number of evaluations are still necessary as the response surface is built in the space of at least 4 dimensions. In the proposed method as shown in the diagram related in Figure 1, Design of Experiments method (DoE) is first used to determine the most sensitive process parameters. Then the response surface is built on the prescribed nodes by the Moving Least Square method and the adaptative approach proposed in (Nayroles, 1991). This response surface is built in the space of dimension number equals to the number of optimization parameters (Belytschko, 1996). The number of the samples is set to be three in each dimension. Then the global minimization is achieved through a genetic algorithm. 


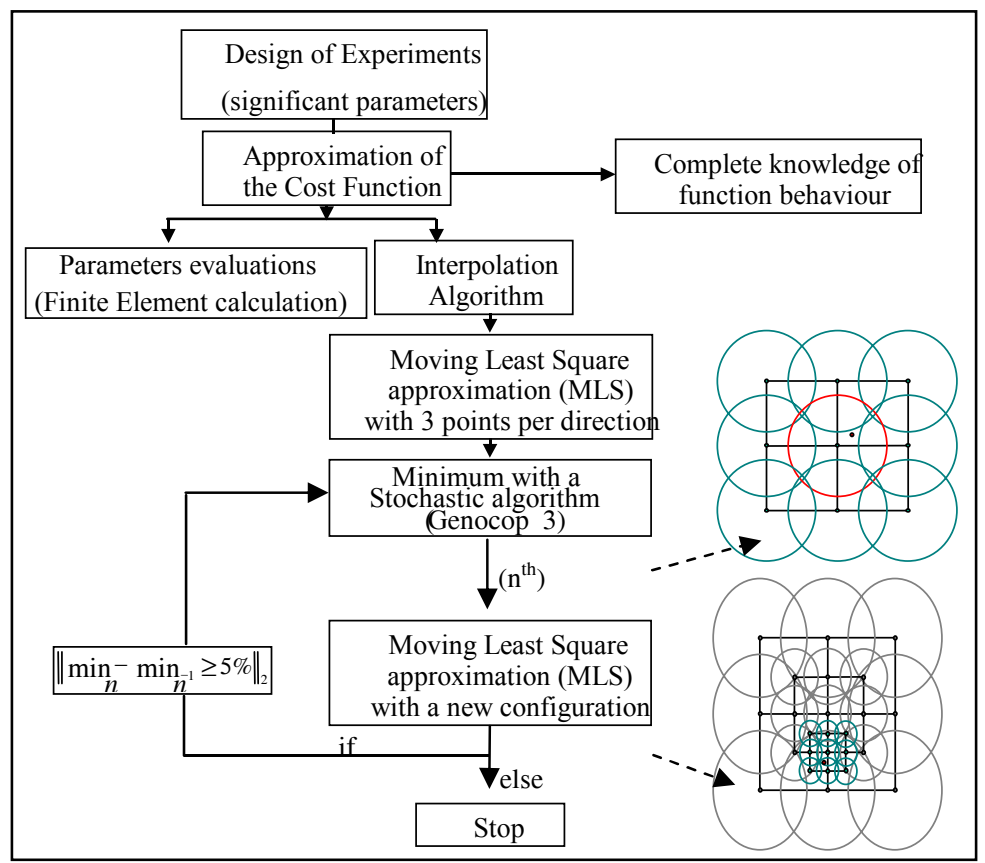

Figure 1. The optimization method for minimization of the segregation in the injection stage associated to powder injection molding

In the next step, an adaptive method is applied. Around the roughly determined minimum position, the response surface is then refined by adding new values issued from the FEM simulations. The refinement is realized by half of the distance between two initial samples (Vergara, 2002). It means that radius of the new sample equals half of the radius of the previous ones, see Figure 1. The main reason to retain the Surface Response Method is associated to the fact one mainly searches a fast and robust solution avoiding local minima and averaging the local variations of the objective function. The second reason is associated to the fact that the computational cost for each simulation remains large.

\subsection{Optimization of the injection stage for a hip implant}

The optimization procedure is applied to the injection of a ceramic hip implant. The process parameters are indicated in Table 1. The variations of filling states in 3D is illustrated in Figure 2a, b, c, d, e. Figure 2e relates the optimized powder volume fraction contours corresponding to $95 \%$ of the filling state. The powder volume fraction varies from 0.38 to 0.42 . 

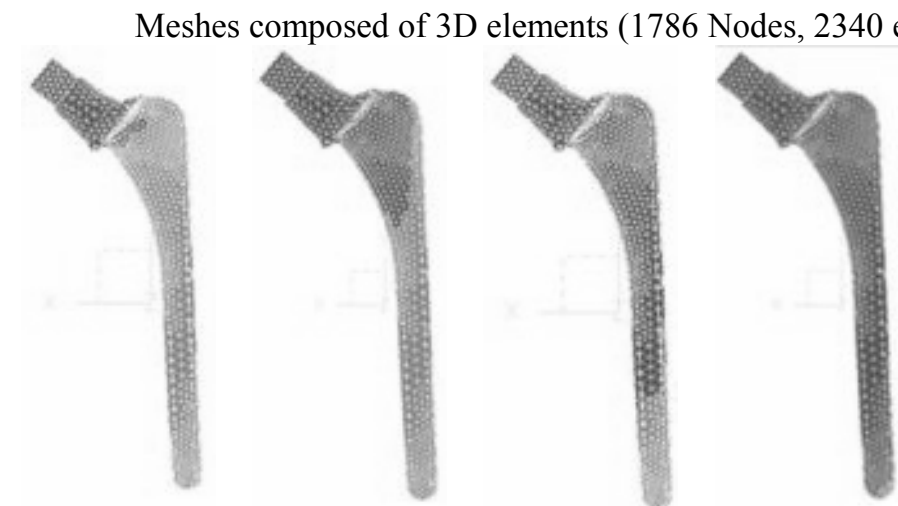
a) $25 \%$
b) $50 \%$
c) $75 \%$
d) $95 \%$
e) powder vol. fraction $(95 \%)$

Figure 2. a), b), c) and d) Evolution of the filling state for different filling ratios. Figure $2 e$ indicates the optimized powder volume fraction corresponding to $95 \%$ of filled state

Then the optimization is launched again in a space with two variables, that corresponds to most sensitive parameters in injection process: the injection pressure $(10-20 \mathrm{MPa})$ and the initial powder volume fraction $(0.4-0.7)$. The position of the samples is illustrated in Figure $3 \mathrm{a}$ and the response surface is shown in Figure $3 \mathrm{~b}$. The optimized solution for both parameters are $\mathrm{P}_{\text {inj }}=20 \mathrm{MPa}$ and $\Phi_{\text {init }}$ $=0.4$, respectively.

Powder volume fraction

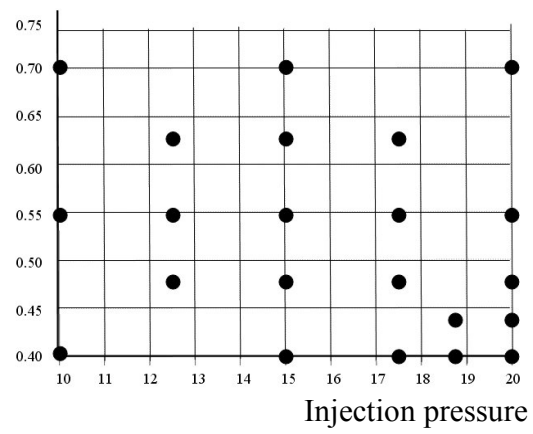

a) Location of the samples after refinement
Objective function

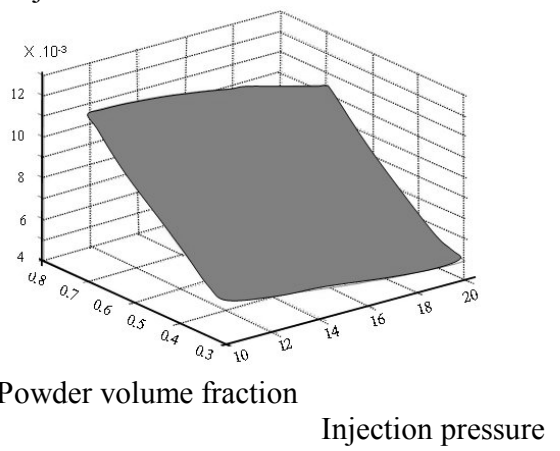

b) Response surface as function of the powder volume fraction and injection pressure

Figure 3. Response surface obtained by the proposed method, a) Powder volume fraction contours, b) Shape of the response surface 
After the injection stage, the debinding one is proceeded to mostly remove the binder. One supposes that the powder volume fraction contours remain unchanged in the debinding stage. This assumption is reasonable, due to the fact that the binder removing leaves the porosity in the parts but it does not change the powder distribution. The contours of powder volume fraction are then handed over to the sintering stage without any significant change. It plays the important role in sintering stage as it may induce the distortion in shape and the defaults in mechanical properties.

\section{Optimization of the sintering stage}

\subsection{Modeling of the material behaviors in sintering}

A linear viscoplastic constitutive law is used to set up the relationship between the stress and strain tensors (Bordia, 1988), expressed as:

$$
\dot{\boldsymbol{\varepsilon}}^{\mathrm{vp}}=\frac{\boldsymbol{\sigma}^{\prime}}{2 G_{\mathrm{p}}}+\frac{\operatorname{tr}(\boldsymbol{\sigma})-3 \sigma_{\mathrm{s}}}{9 K_{\mathrm{p}}} \mathbf{I}
$$

where $\dot{\boldsymbol{\varepsilon}}^{\mathrm{vp}}$ is the viscoplastic strain rate, $\boldsymbol{\sigma}^{\prime}$ is the deviatoric stress tensor, $\operatorname{tr}(\boldsymbol{\sigma})$ is the trace of stress tensor, $G_{\mathrm{p}}$ is the shear viscosity modulus, $K_{\mathrm{p}}$ is the bulk modulus, $\sigma_{\mathrm{s}}$ is the sintering stress and $\mathbf{I}$ is the second order identity tensor.

Different models have been developed to calibrate the constitutive law; the following expression is used in the present analysis (Bordia, 1988):

$$
G_{\mathrm{p}}=\frac{\eta_{\mathrm{z}}}{2\left(1+v_{\mathrm{vp}}\right)}, K_{\mathrm{p}}=\frac{\eta_{\mathrm{z}}}{3\left(1-2 v_{\mathrm{vp}}\right)}, v_{\mathrm{vp}} \approx \frac{1}{2} \sqrt{\frac{\rho}{3-2 \rho}}
$$

in which $\eta_{\mathrm{z}}$ and $v_{\mathrm{vp}}$ are respectively the axial viscosity and viscous Poisson ratio of the porous material, $\rho$ is the apparent density of the porous material. The axial viscosity can be expressed in the following form (Song et al., 2006):

$$
\eta_{\mathrm{z}}=\frac{k T G^{3} \rho^{2}}{\Omega D_{\mathrm{b} 0} \exp \left(-Q_{\mathrm{b}} / R T\right)}
$$

where $k$ is a constant, $T$ is the absolute temperature, $G$ represents the grain size, $\Omega$ is the atomic or molecular volume, $D_{\mathrm{b} 0}$ is the grain boundary coefficient diffusion on, $Q_{\mathrm{b}}$ is the activation energy for grain boundary diffusion and $R$ is the gas constant.

The sintering stress corresponding to the ceramic powder is expressed in the following form (He, 2005): 


$$
\sigma_{\mathrm{s}}=\frac{6\left(2 \gamma_{\mathrm{sv}}-\gamma_{\mathrm{ss}}\right)}{G} \rho^{2}\left(\frac{2 \rho-\rho_{0}}{1-\rho_{0}}\right)
$$

where $\gamma_{\mathrm{sv}}$ is the surface energy, $\gamma_{\mathrm{ss}}$ is the grain boundary energy, and $\rho_{0}$ is the initial relative density. The following equation has been chosen for the powder particles growth for alumina powder mixture (Kang, 2004):

$$
\frac{d G}{d t}=\frac{110 \delta_{\mathrm{s}} D_{\mathrm{s} 0} \exp \left(-Q_{\mathrm{s}} / R T\right) \gamma_{\mathrm{ss}} \Omega}{k T G^{3}}(1-\rho)^{-\frac{4}{3}}
$$

where $\delta_{\mathrm{s}}$ is the surface diffusion thickness, $D_{\mathrm{s} 0}$ represents surface diffusion frequency, and $Q_{\mathrm{s}}$ is the activation energy associated to surface diffusion.

\subsection{Identification of the constitutive law for solid state sintering}

For the purpose of sintering simulation, an identification algorithm has been applied to determine the material parameters in the sintering constitutive model. An optimization strategy is proposed to minimize the gaps between the shrinkage curves obtained from dilatometer experiments and by numerical simulations, respectively.

The material parameters are the variables to be optimized, so the optimization problem is formulated as a minimization one expressed as:

$$
\min F(x)=\sum_{i=1}^{n}\left|f_{e}\left(T_{i}, x\right)-f_{s}\left(T_{i}, x\right)\right|^{2} \text { with } x=\left[Q_{\mathrm{b}}, \delta_{\mathrm{b}} D_{\mathrm{b} 0}, Q_{\mathrm{s}}, \delta_{\mathrm{s}} D_{\mathrm{s} 0}\right]
$$

where $f_{e}\left(T_{i}, x\right)$ is the shrinkage curve issued from the dilatometer experiments, $f_{s}\left(T_{i}, x\right)$ is the shrinkage curve obtained by numerical simulations and $x$ is the set of variables for material parameters. In the sintering model for alumina powder, as shown in Equation [3-6], the parameters $k, R$ and $\Omega$ are physical constants. The surface energy $\gamma_{\mathrm{sv}}$ is chosen equal $0.71 \mathrm{~J} / \mathrm{m}^{2}$, the grain boundary energy $\gamma_{\mathrm{ss}}$ is set to be $0.34 \mathrm{~J} / \mathrm{m}^{2}$ (Kang, 2004). So the identification procedure consists to find the optimal values corresponding to $Q_{\mathrm{b}}, \delta_{\mathrm{b}} D_{\mathrm{b} 0}, Q_{\mathrm{s}}$ and $\delta_{\mathrm{s}} D_{\mathrm{s} 0}$. The identification procedure for sintering parameters is applied to the powder alumina and dissolvable binder mixture. The shrinkage curve vs. sintering time has been measured in our laboratory with a dilatometer. The resulting identified parameters are given in Table 2. These parameters are compared with these ones issued from literature (Kang, 2004). The dilatometer test results and the simulations with the initial and identified parameters are shown in Figure 4. 
Table 2. The identified parameters and their values related in literature

\begin{tabular}{lccccc}
\hline & $\begin{array}{c}Q_{\mathrm{b}} \\
(\mathrm{kJ} / \mathrm{mol})\end{array}$ & $Q_{\mathrm{s}}(\mathrm{kJ} / \mathrm{mol})$ & $\delta_{\mathrm{b}} D_{\mathrm{b} 0}\left(\mathrm{~m}^{3} / \mathrm{s}\right)$ & $\delta_{\mathrm{s}} D_{\mathrm{s} 0}\left(\mathrm{~m}^{3} / \mathrm{s}\right)$ \\
\hline Reference $(\mathrm{Kang}, 2004)$ & 418 & 493 & $8.6 \times 10^{-10}$ & $1.26 \times 10^{-7}$ \\
\hline Heating rate $20{ }^{\circ} \mathrm{C} / \mathrm{min}$ & 351.09 & 234.67 & $2.1911 \mathrm{e}^{-9}$ & $4.5284 \mathrm{e}^{-12}$ \\
\hline holding at $1600{ }^{\circ} \mathrm{C}$ & 358.68 & 380.97 & $2.4503 \mathrm{e}^{-9}$ & $3.8097 \mathrm{e}^{-8}$ \\
\hline & & & & & \\
\hline
\end{tabular}

Figure 4. The shrinkage obtained from dilatometer tests and simulation results obtained from identified parameters or coming from (Kang, 2004)

\subsection{The application to a hip implant}

An example of hip implant has been realized in our laboratory (Liksonov, 2006), in order to validate the overall identification optimization process. The contours of the initial density for sintering simulation are issued from the optimization of injection process on the basis of biphasic simulation, see Paragraph 2. The mean density associated to the green part corresponding to the hip implant is equal to 0.58 . The sintering parameters of the model have been identified and are related in Table 2. The initial mesh for the sintering simulation is shown in Figure 5a. The mean shrinkage that results from simulation corresponds to $11.85 \%$. The final density reaches a value $84.5 \%$ issued in simulation. The shape of sintered hip implant is shown in Figure 5a, too. The measurements on the hip implants indicate that the shrinkage is about $15.4 \%$, and the final density $95 \%$ is obtained. The values resulting from experiments are greater than the ones obtained by simulation. This significant difference is due to the fact that the sintering model is sometimes not adequate for prediction of the density evolution at the last stage of sintering by solid state diffusion. Nevertheless the model is correct for simulation of the first stages in sintering process but not up to the last stage. 


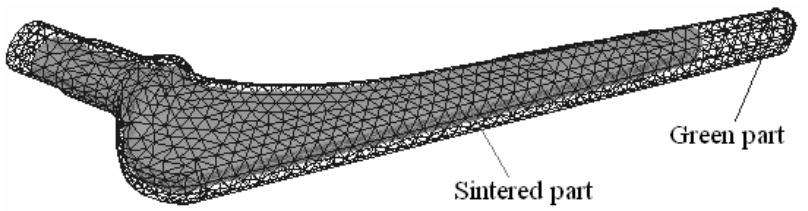

(a) Initial mesh (debinded part) and final size (sintered hip implant)

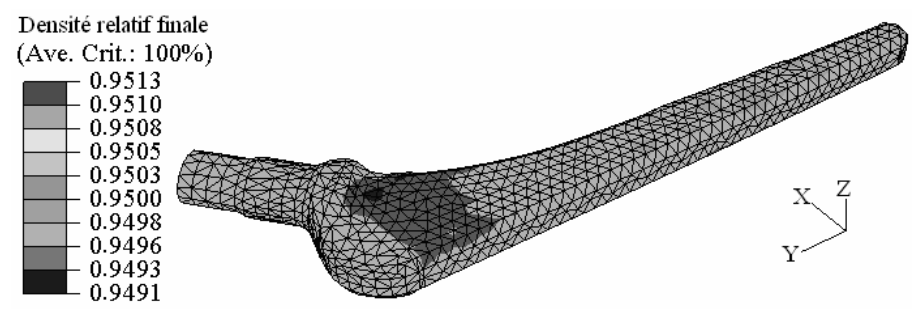

(b) Density contours in the sintered hip implant

Figure 5. Mesh and density contours resulting from the sintering of the hip implant

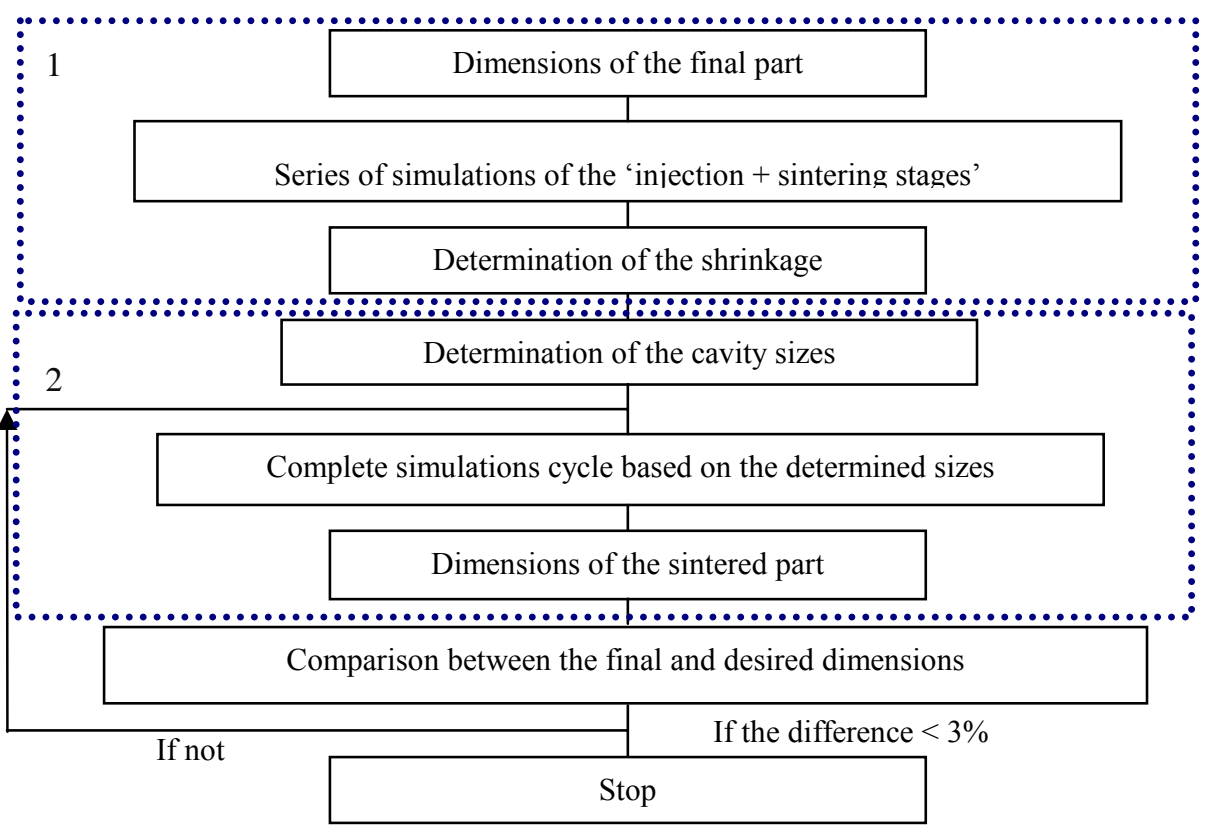

Figure 6. The mold cavities dimensions resulting from the overall optimization of the PIM process 


\subsection{Optimisation of the complete processing cycle}

The objective of the overall optimization proposed strategy is to get the final dimensions and material properties of the sintered parts. It means that the improvements from each optimization step are aimed to upgrade and update the final results. In the backward sense, the parameters for the injection phase should be guided to provide the suitable initial conditions for the sintering stage to get the prescribed dimensions and material properties. Based on the powder volume fraction issued from the injection stage, the shrinkage predicted by sintering simulation should match the required final dimensions. Then, it is realistic to determine the sizes of mold cavities according to the required final dimensions, sintering shrinkage, and the powder volume fraction after the injection. Once a cycle of the optimization is realized to get the predicted final dimensions, the difference between the required and predicted final parts is reported to launch again a complete improvements cycle. This procedure continues up to the overall optimization, until the prescribed tolerance is achieved, see the diagram in Figure 6. The method that is used consists to choose a fixed point in the mesh and to apply a scaling from this fixed node with the ratio corresponding to the last obtained mean dimensional shrinkage.

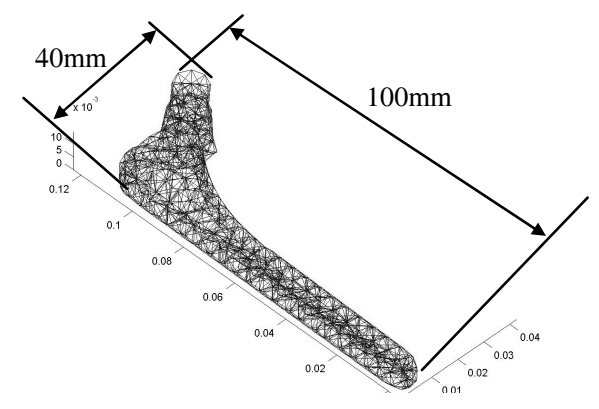

Figure 7. Geometry of the required part resulting from PIM processing

In the section below, the sequential optimization for the final form is applied to the hip implant example. At the end of PIM process, the following geometry is required for the final sintered parts, see Figure 7. The results of proposed optimization strategies are shown in Figure 8. After 3 iterations, the shrinkage reach a stabilized value equal $15.2 \%$ that permits to determine the dimensions of mold cavity. The optimization of the overall process steps permits to evaluate the size of mold cavities. The uniform distribution of powder phase after injection is also improved to satisfy the final dimension requirements. The macroscopic sintering model parameters should be calibrated from experiments to evaluate the final shrinkage in sintering process with the required accuracy. 


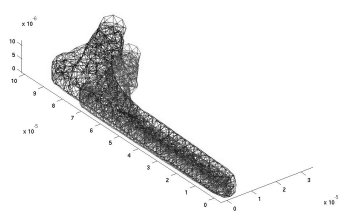

$1^{\text {st }}$ iteration :

- Simulation of injection with initial size

- Simulation of sintering process

- Result: $15.5 \%$ of shrinkage

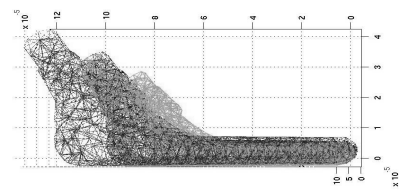

$2^{\text {nd }}$ iteration :

- Geometric growing factor 1.1150

- Simulation of injection + sintering

$-15.21 \%$ of shrinkage

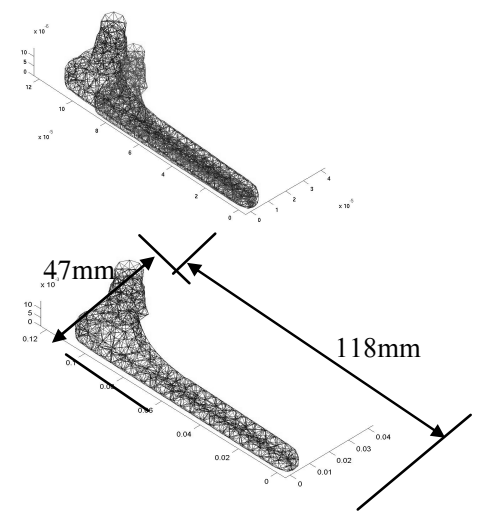

$3^{\text {rd }}$ iteration :

- Geometric growing factor 1.1521

- Simulation of injection + sintering

$-15.2 \%$ of shrinkage

- Dimensions of the mold cavity

- The computation for 3 iterations take 54 hours (on a standard PC)

Figure 8. Optimization of the final hip implant corresponding to $\mathrm{Al}_{2} \mathrm{O}_{3}$ mixed powders

\section{Conclusions}

An overall optimization strategy for PIM process has been developed and applied to the determination of the mold cavities geometry and processing conditions. The approach combines sequentially the optimization of the injection moulding stage followed by the sintering one. The solver that is used for the injection stage is based on a biphasic approach and the optimization strategy based on a Response Surface Method leads to the minimization of the powder segregation effects. Then the overall optimization includes an inverse identification of the solid state sintering parameters, followed by a solver for prediction of the shrinkage resulting from the sintering stage. The later is capable to model more precisely the physical mechanisms associated to the sintering process, and to predict the final s dimensions of the sintered parts. Finally, based on the optimization of injection moulding stage and sintering one, the optimization loop leads to the appropriate mould cavity geometry as well as sintering 
parameters to get the component with the required geometry. An example of hip implant made of the alumina powder and dissolvable binder has been realized to demonstrate the efficiency of the proposed procedure. According to the final dimensions and identified model for sintering, it is realistic to determine the size of initial mold cavities and minimize the shape distortion by minimization of the powder segregation in injection.

\section{References}

Ayad G., Barriere T., Gelin J.C., "Optimization of powder segregation occurring in metal injection molding of stainless steels", Int. J. of Forming Process, vol. 9, n 1, 2006, p. 9-28.

Barriere T., Physique et Technologie du Moulage par Injection de Poudres, Mémoire d'HDR, Université de Franche-Comté, décembre 2005, p. 1-214.

Barriere T., Gelin J.C., Liu B., "Experimental and numerical analyses of powder segregation on the properties and quality of parts produced by MIM", Powder Metall., vol. 44, n 3, 2001, p. 228-234.

Belytschko T., Krongauz Y., Organ D., Fleming M., Krysl P., "Meshless methods: An overview and recent developments", Comput. Methods Appl. Mech. Engrg., vol. 139, 1996, p. 3-47.

Bordia R.K., Scherer G.W., "On constrained sintering-I Constitutive model for a sintering body", Acta Metall., vol. 36, n 9, 1988, p. 2393-2397.

Gelin J.C., Barriere T., «Ségrégation de phases dans les écoulements de polymères fondus chargés en poudres métalliques », Mécanique et Industries, Ed. by EDP Sciences, vol. 5, $n^{\circ} 4,2004$, p. 429-440.

German R.M., Bose A., Injection moulding of metal and ceramics, Princeton, MPIF, 1997.

He Z., Ma J., "Constitutive modeling of alumina sintering: grain-size effect on dominant densification mechanism", Comp. Mater. Sci., vol. 32, 2005, p. 196-202.

Kang S-J.L., Kang Y.I., "Sintering kinetics at final stage sintering: model calculation and map construction", Acta Mater., vol. 52, 2004, p. 4573-4578.

Liksonov D., Experimental and numerical study of the manufacturing and behavior of the formal THR components produced by injection molding of fiber composite and by powder technologies, Ph. D Thesis, University of Franche-Comté, 2006.

Nayroles B., Touzot G., Villon P., La Méthode des Eléments Diffus, Comptes Rendus de l'Academie de Sciences, 313, série II, Paris, France, 1991, p. 133-138.

Song J., Gelin J.C., Barriere T., Liu B., "Experiments and numerical modelling of solid state sintering for 316L stainless steel components", J. Mater. Process. Technol., vol. 177, 2006, p. 352-355.

Vergara F.E., Khouja M., Michalewicz Z., “An evolutionary algorithm for optimisation material flow in supply chains", Computers \& Industrial Engineering, vol. 3, 2002, p. 407-421. 
